In the book: Inverse problems, tomography and image processing, Plenum publishers, New York, 1998, pp. 1-8 (ed. A.G.Ramm).

\title{
INVERSE ACOUSTIC SCATTERING BY A LAYERED OBSTACLE
}

\author{
C. Athanasiadis ${ }^{1}$, A. G. Ramm ${ }^{2}$ and I. G. Stratis ${ }^{1}$ \\ ${ }^{1}$ University of Athens, Department of Mathematics, \\ Panepistimiopolis, GR 15784 Athens, Greece. \\ e-mail: istratis@atlas.uoa.gr \\ ${ }^{2}$ Department of Mathematics, Kansas State University, Manhattan, \\ KS 66506-2602, U.S.A. \\ e-mail: ramm@math.ksu.edu
}

\begin{abstract}
A uniqueness theorem is proved for the inverse acoustic scattering problem for a piecewise-homogeneous obstacle under the assumption that the scattering amplitude is known for all directions of the incident and the scattered field at a fixed frequency.
\end{abstract}

\section{Introduction}

The inverse problem of the scattering of a plane acoustic wave by a multilayered scatterer is studied in this work. Such a scatterer consists of a finite number of homogeneous layers, and is described in detail in Section 2. The corresponding direct scattering problem has been studied by Athanasiadis and Stratis, who have shown existence and uniqueness of its solution in [2], and have implemented the low frequency theory for the determination of the solution in [1]. Alternative formulation of a similar problem for the impedance equation has been studied in [18] and references therein. In the present work uniqueness of the solution to inverse scattering problem is established provided that the scattering amplitude is known for all directions of incident and scattered fields at a fixed frequency. The proof is based on 
an orthogonality result and is similar to the proofs in [7]-[12]. Similar ideas have been applied in [12] to some inverse scattering problems, inverse spectral problems, inverse geophysical problems, and some other inverse problems. The actual reconstruction of the scatterer is still an open problem. There is a number of papers in which the surface of an acoustically soft obstacle is found numerically from the scattering data, but these contain parameter-fitting schemes, rather than a reconstruction procedure based on an analytical or numerical inversion method, and they give no error estimate for the solution. Stability estimates for the recovered obstacle are not given in the present work. They were given in the papers [13], [14] for the case of a non-stratified soft scatterer.

\section{The Direct Problem}

Let $D$ be a bounded closed subset of $\mathbb{R}^{3}$ with boundary $S_{0}$. The set $D$ is divided into annuli-like regions $D_{m}$ by surfaces $S_{m}$, where $S_{m-1}$ surrounds $S_{m}$, and $S_{m-1} \cap S_{m}=\emptyset, m=1,2, \ldots, M$. The $S_{m}, m=0,1, \ldots, M$ are assumed to be $C^{1, \lambda}$-surfaces, $\lambda \in(0,1]$. Moreover we assume that the origin is contained in $D_{M+1}$, the "last" region. The exterior $D_{0}$ of $D$, as well as each of $D_{m}$, are homogeneous isotropic media of mass density $\rho_{m}$ and compressibility $\gamma_{m}$. The wave number $k_{m}$ in each region $D_{m}$ is expressed in terms of the free space $\left(D_{0}\right)$ wave number $k_{0}>0$, by the relation

$$
k_{m}^{2}=\frac{\gamma_{0}}{\gamma_{m}} \frac{\rho_{m}}{\rho_{0}} k_{0}^{2}
$$

We assume that a plane acoustic wave $u^{\text {inc }}$ is incident upon $D$; suppressing the harmonic time dependence $\exp (-i \omega t), \omega$ being the angular frequency, we have

$$
u^{\text {inc }}\left(x, \alpha, k_{0}\right)=e^{i k_{0} x \cdot \alpha},
$$

where $\alpha$ is the unit vector in the direction of propagation.

The total exterior acoustic field $u_{0}$ is given by the formula:

$$
u_{0}\left(x, \alpha, k_{0}\right)=u^{\mathrm{inc}}\left(x, \alpha, k_{0}\right)+u^{\mathrm{sc}}\left(x, \alpha, k_{0}\right)
$$

where $u^{\mathrm{sc}}$ is the scattered field given by

$$
u^{\mathrm{sc}}\left(x, \alpha, k_{0}\right)=A\left(\alpha^{\prime}, \alpha, k_{0}\right) \frac{e^{i k_{0} r}}{r}+o\left(\frac{1}{r}\right), r \rightarrow \infty
$$

with $r=|x|$ and $\alpha^{\prime}=\frac{x}{r}$. $A\left(\alpha^{\prime}, \alpha, k_{0}\right)$ is the scattering amplitude [6]. Formula (2.4) implies the radiation condition for the scattered field. 
Let $u_{m}$ be the acoustic field in $D_{m}$. Then the problem of scattering of $u^{\text {inc }}$ by the multi-layered scatterer $D$, is described by the following transmission problem:

Find $u_{m} \in C^{2}\left(D_{m}\right) \cap C\left(\overline{D_{m}}\right)$, such that

$$
\left.\begin{array}{c}
\Delta u_{m}+k_{m}^{2} u_{m}=0, \quad \text { in } \quad D_{m}, m=0,1, \ldots, M+1 \\
u_{m+1}-u_{m}=0 \\
\frac{1}{\rho_{m+1}} \frac{\partial u_{m+1}}{\partial \nu}-\frac{1}{\rho_{m}} \frac{\partial u_{m}}{\partial \nu}=0
\end{array}\right\}, \quad \text { on } \quad S_{m}, m=0,1, \ldots, M
$$

along with (2.3) and (2.4).

It has been proved in [2] that this transmission problem has a unique solution.

Remark 2.1 The fundamental solution

$$
\Phi(x, y)=\frac{e^{i k|x-y|}}{4 \pi|x-y|}
$$

of the Helmholtz equation $\Delta u+k^{2} u=0$ satisfies

$$
\operatorname{grad}_{x} \Phi(x, y)=\left(i k-\frac{1}{|x-y|}\right) \Phi(x, y) \frac{x-y}{|x-y|}
$$

and the asymptotic formulas

$$
\Phi(x, y)=\frac{1}{4 \pi|x-y|}[1+O(|x-y|)],|x-y| \rightarrow 0
$$

and

$$
\operatorname{grad}_{x} \Phi(x, y)=\frac{x-y}{4 \pi|x-y|^{3}}\left[1+O\left(|x-y|^{2}\right)\right],|x-y| \rightarrow 0 .
$$

If $\Phi_{m}(x, y)$ is the fundamental solution of $\Delta u+k_{m}^{2} u=0$ in $D_{m}, m=$ $0,1, \ldots, M+1$, then $\Phi_{m}(x, y)$ satisfies (2.9) and (2.10). The asymptotic behavior for elliptic operators with smooth coefficients was derived by the parametrix methods in the theory of pseudodifferential operators and also by classical methods (see [5]).

We will need also the asymptotics of the principal part of the fundamental solution to elliptic equations of the second order of the divergent form (see (3.1) with $q(x)=0$ below) for piecewise-smooth $a(x)$. We assume that $a(x)$ has a smooth surface $S$ as a discontinuity surface, and $a(x)$ together with its derivatives is continuous up to $S$ from inside and outside. In this case one can assume without loss of generality that $S$ is a plane, say the plane $x_{n}=0$, 
and the function $a(x)$ is constant $a_{+}$if $x_{n}>0$, and $a(x)=a_{-}$if $x_{n}<0$. The fundamental solution $g$ is assumed continuous across $S$, and $a \frac{\partial g}{\partial \nu}$ is continuous across $S$, where $\nu$ is the normal to $S$, in our case $\nu$ is directed along $x_{n}$. In [17] a formula for $g(x, y)$ is obtained, where $g(x, y)$ satisfies the above transmission conditions across $S$ and the differential equation $\operatorname{div}[a(x) \operatorname{grad} g]+k_{0}^{2} q(x) g=$ $-\delta(x-y)$ in $\mathbb{R}^{n}$. If $a(x)$ is piecewise-constant, then a global formula for $g$ is given in [17], see formulas (2.11) and (2.12) below. In [4], it is claimed that $g$ is of the form $c|x-y|^{-1}$ if $n=3$, where $c=\left[\left(a_{+}+a_{-}\right)(2 \pi)\right]^{-1}$. This claim is not correct and we give below the correct formula. However, if $y$, the pole of $g(x, y)$, belongs to the interface $S$, then, as $|x-y| \rightarrow 0$, one obtains $g \sim c|x-y|^{-1}$, with $c$ defined above as in [4].

Let $\gamma:=\left(a_{+}-a_{-}\right) /\left(a_{+}+a_{-}\right), \rho:=|\hat{x}-\hat{y}|, \hat{x}:=\left(x_{1}, \ldots, x_{n-1}\right), A_{ \pm}:=$ $\left(4 \pi a_{ \pm}\right)^{-1}, r:=|x-y|$. We claim that the following global formula holds

$$
\begin{aligned}
& g=A_{+}\left[\frac{1}{r}+\frac{\gamma}{\left(\rho^{2}+\left[\left|x_{3}\right|+\left|y_{3}\right|\right]^{2}\right)^{1 / 2}}\right], y_{3}>0, \\
& g=A_{-}\left[\frac{1}{r}-\frac{\gamma}{\left(\rho^{2}+\left[\left|x_{3}\right|+\left|y_{3}\right|\right]^{2}\right)^{1 / 2}}\right], y_{3}<0 .
\end{aligned}
$$

The idea of the proof of formulas $(2.11)-(2.12)$ is simple: one Fouriertransforms the equation for $g$ with respect to transversal variables $\hat{x}$ and gets an ordinary differential equation for the function $\widetilde{g}$, the Fourier transform of the above $g$. The solution to this equation with the transmission conditions at $x_{3}=0$ leads to the formula:

$$
\begin{aligned}
& \tilde{g}=\frac{\exp \left(-\xi\left|x_{3}-y_{3}\right|\right)}{2 \xi a_{+}}+\gamma \frac{\exp \left(-\xi\left[\left|x_{3}\right|+\left|y_{3}\right|\right]\right)}{2 \xi a_{+}}, y_{3}>0, \\
& \widetilde{g}=\frac{\exp \left(-\xi\left|x_{3}-y_{3}\right|\right)}{2 \xi a_{-}}-\gamma \frac{\exp \left(-\xi\left[\left|x_{3}\right|+\left|y_{3}\right|\right]\right)}{2 \xi a_{-}}, y_{3}<0 .
\end{aligned}
$$

Inverse transforming (2.14) with respect to $\xi$-variable, one gets $(2.11),(2.12)$. Let us note that (2.11), (2.12) hold locally for equations with variable coefficients.

Remark 2.2 The assumption that the $S_{m}, m=0,1, \ldots, M$ are of class $C^{1, \lambda}$, $\lambda \in(0,1]$, may be weakened to $S_{m}$ being Lipschitz surfaces (see [15], [16] ). 


\section{Uniqueness of Solutions to the Inverse Prob- lem}

We start this section by noticing that (2.5) may be written in the following unified way

$$
(L u)(x):=\operatorname{div}[a(x) \operatorname{grad} u(x)]+k_{0}^{2} q(x) u(x)=0, \text { in } \bigcup_{m=0}^{M+1} D_{m},
$$

where

$$
\begin{aligned}
& u_{m}(x)=u(x) \chi_{D_{m}}(x), m=0,1, \ldots, M+1 \\
& a(x)= \begin{cases}1, & x \in D_{0} \\
\frac{1}{\rho_{m}}, & x \in D_{m} \cup S_{m-1}, m=1,2, \ldots, M+1\end{cases} \\
& q(x)= \begin{cases}1, & x \in D_{0} \\
\frac{1}{\rho_{m}} \frac{k_{m}^{2}}{k_{0}^{2}}, & x \in D_{m} \cup S_{m-1}, m=1,2, \ldots, M+1,\end{cases}
\end{aligned}
$$

$\chi_{D_{m}}(x)$ being the characteristic function of $D_{m}$. Using the notation $[h(x)]=$ $h^{+}(x)-h^{-}(x), h^{+}(x)\left(h^{-}(x)\right)$ denoting the limit of $h$ on $S$ from the exterior (interior) of $S$, we may write (2.6) as

$$
[u]=0 \quad \text { and } \quad\left[a \frac{\partial u}{\partial \nu}\right]=0 \text {, on } S_{m} .
$$

Moreover, in view of (2.3) we have

$$
u(x)=u^{\mathrm{inc}}(x)+u^{\mathrm{sc}}(x),
$$

while $u^{\text {sc }}$ is assumed to satisfy the Sommerfeld radiation condition

$$
\lim _{r \rightarrow \infty} r\left(\frac{\partial u^{\mathrm{sc}}}{\partial r}-i k_{0} u^{\mathrm{sc}}\right)=0
$$

uniformly in all directions $\frac{x}{r}$.

Therefore, the direct transmission problem (2.3)-(2.6) may be written as the problem consisting of (3.1), (3.5), (3.6), (3.7), and will be denoted by (DTP). Now, let

$$
\mathcal{H}:=H^{1}(D) \cap\left\{h \in H^{1}\left(\overline{D_{0}}{ }_{l o c}\right): h \text { satisfies (3.6) and }(3.7)\right\} .
$$

We denote by $H_{0}$ the set of $h$ which satisfy (3.8) with the condition that $h$ solves (3.6) dropped and (3.7) replaced for the elements of $H_{0}$ by the condition that $h$ vanishes near infinity. For the use of such spaces we refer to [3]. We are now in a position to give 
Definition 3.1 (DTP) has a weak solution $u \in \mathcal{H}$ iff

$$
\int_{\mathbb{R}^{3}}\left\{-a(x) \operatorname{grad} u(x) \cdot \operatorname{grad} v(x)+k_{0}^{2} q(x) u(x) v(x)\right\} d x=0, \forall v \in H_{0} .
$$

Let us note that the transmission conditions (3.5) are incorporated in (3.9).

The inverse transmission problem consists of finding $D$ (i.e. the surfaces $\left.S_{m}, m=0,1, \ldots, M\right)$, given the scattering amplitude $A\left(\alpha^{\prime}, \alpha, k_{0}\right)$ for all directions of incident and scattered fields at a fixed $k_{0}>0$. The constant parameters $k_{m}^{2}$ and $\rho_{m}$ of the $m$-th layer are assumed known but the boundaries of the layers are not known when the inverse problem is studied.

In this paper we study the uniqueness of the solutions to the inverse transmission problem. The main uniqueness result is proved by an approach similar to that used in [7]- [12].

For the formulation of the results, we assume that there are two multilayered scatterers $D^{(1)}$ and $D^{(2)}$. Each $D^{(j)}$ is stratified by surfaces $S_{m}^{(j)}$ into layers $D_{m}^{(j)}, m=0,1, \ldots, M$ and $j=1,2$, as described in Section 2 .

We start with the following orthogonality result.

Proposition 3.1 Let us assume that

$$
A^{(1)}\left(\alpha^{\prime}, \alpha, k_{0}\right)=A^{(2)}\left(\alpha^{\prime}, \alpha, k_{0}\right)
$$

for all $\alpha^{\prime}$, $\alpha$ on the unit sphere, and a fixed $k_{0}>0$. Let $w_{j}$ be the solution of $L^{(j)} w_{j}=0$ in $\Omega, j=1,2$, satisfying (3.6)-(3.7), where $\Omega$ is any domain containing $\overline{D^{(1)}} \cup \overline{D^{(2)}}$. Then

$$
\begin{aligned}
& \int_{D^{(1)}}\left\{-a_{1}(x) \operatorname{grad} w_{1}(x) \cdot \operatorname{grad} w_{2}(x)+k_{0}^{2} q_{1}(x) w_{1}(x) w_{2}(x)\right\} d x= \\
& \int_{D^{(2)}}\left\{-a_{2}(x) \operatorname{grad} w_{1}(x) \cdot \operatorname{grad} w_{2}(x)+k_{0}^{2} q_{2}(x) w_{1}(x) w_{2}(x)\right\} d x
\end{aligned}
$$

Proof. Let $w:=w_{1}-w_{2}, a:=a_{1}-a_{2}$, and $q:=q_{1}-q_{2}$. Subtract from (3.1) with $w=w_{1}, a=a_{1}, q=q_{1}$ this equation with $w=w_{2}, a=a_{2}, q=q_{2}$, to get

$$
\operatorname{div}\left(a_{1} \operatorname{grad} w\right)+k_{0}^{2} q_{1} w=-\operatorname{div}\left(a \operatorname{grad} w_{2}\right)-k_{0}^{2} q w_{2} .
$$

Let $\Omega_{0}:=\mathbb{R}^{3} \backslash\left(\overline{D^{(1)}} \cup \overline{D^{(2)}}\right)$. We have

$$
\Delta w+k_{0}^{2} w=0, \text { in } \Omega_{0} .
$$

From (3.10), (2.3), (2.4) we derive that

$$
w(x)=O\left(\frac{1}{|x|^{2}}\right),|x| \rightarrow \infty,
$$


whereby

$$
w(x)=0 \text { for }|x| \text { large. }
$$

Hence, by the unique continuation principle, we obtain

$$
w(x)=0, \quad x \in \Omega_{0} .
$$

Multiplying (3.12) by $\varphi \in H_{0}^{1}(\Omega)$ and integrating over $\Omega$ we get

$$
\int_{\Omega}\left\{-a_{1} \operatorname{grad} w \cdot \operatorname{grad} \varphi+k_{0}^{2} q_{1} w \varphi\right\} d x=\int_{\Omega}\left\{a \operatorname{grad} w_{2} \cdot \operatorname{grad} \varphi-k_{0}^{2} q w_{2} \varphi\right\} d x
$$

Now, let $\Omega_{*}$ be any open bounded set in $\mathbb{R}^{3}$, containing $D^{(1)} \cup D^{(2)}$, such that $\bar{\Omega}_{*} \subset \Omega$. Let $\psi \in C_{0}^{\infty}(\Omega), \psi=1$ in $\Omega_{*}$. Then $\varphi:=\psi w_{1} \in H_{0}^{1}(\Omega)$. Hence

$$
\begin{aligned}
& \int_{\Omega}\left\{-a_{1} \operatorname{grad} w \cdot \operatorname{grad} \varphi+k_{0}^{2} q_{1} w \varphi\right\} d x= \\
& \int_{\Omega}\left(L^{(1)} \varphi\right) w d x=\int_{\Omega}\left(L^{(1)} w_{1}\right) w d x=0,
\end{aligned}
$$

and so

$$
\int_{\Omega}\left\{a \operatorname{grad} w_{2} \cdot \operatorname{grad} \varphi-k_{0}^{2} q w_{2} \varphi\right\} d x=0 .
$$

But in $\Omega_{0}$ we have $a=0, q=0$, while in $D^{(1)} \cup D^{(2)}$ we may take $\varphi=w_{1}$, whereby (3.11) follows.

We now prove:

Theorem 3.1 If (3.10) holds, then $D^{(1)}=D^{(2)}$.

Proof. As mentioned in the description of the inverse transmission problem, it suffices to show that $S_{m}^{(1)}$ coincides with $S_{m}^{(2)}, m=0,1, \ldots, M$. Suppose, to the contrary, that there is a $m_{0} \in\{0,1, \ldots, M\}$ such that $S_{m_{0}}^{(1)}$ does not coincide with $S_{m_{0}}^{(2)}$. Let $\widetilde{D}^{(j)}, j=1,2$, denote the domain circumscribed by $S_{m_{0}}^{(j)}$. Assume that $\widetilde{D}^{(1)}$ is not contained in $\widetilde{D}^{(2)}$. Without loss of generality, we suppose that $m_{0}=0$ (and hence $\widetilde{D}^{(j)}=D^{(j)}$ ). Let $z$ be on the part of $S_{0}^{(1)}$ which is not inside $D^{(2)}$. Consider a ball $B_{z}$, centered at $z$, having no common points with $\overline{D^{(2)}}$, while its part inside $D^{(1)}$ lies in the first layer $D_{1}^{(1)}$, without touching $S_{1}^{(1)}$. Let $w_{j}(x, y)$ be the Green function of $L^{(j)}$, and $y \in \Omega_{0}$. Since the Green function with $y$ away from $B_{z}$ can be approximated with arbitrary accuracy in $H^{2}\left(B_{z}\right)$ by scattering solutions (see [12], [9]), one can use as $w_{j}$ in (3.11) the functions $w_{j}(x, y)$. Write (3.11) as

$$
\begin{aligned}
& \int_{D^{(1)} \cap B_{z}}\left\{-a_{1}(x) \operatorname{grad} w_{1}(x, y) \cdot \operatorname{grad} w_{2}(x, y)+k_{0}^{2} q_{1}(x) w_{1}(x, y) w_{2}(x, y)\right\} d x= \\
= & \int_{D^{(1)} \backslash B_{z}}\left\{a_{1}(x) \operatorname{grad} w_{1}(x, y) \cdot \operatorname{grad} w_{2}(x, y)-k_{0}^{2} q_{1}(x) w_{1}(x, y) w_{2}(x, y)\right\} d x+ \\
+ & \int_{D^{(2)}}\left\{-a_{2}(x) \operatorname{grad} w_{1}(x, y) \cdot \operatorname{grad} w_{2}(x, y)+k_{0}^{2} q_{2}(x) w_{1}(x, y) w_{2}(x, y)\right\} d x \cdot(3.20)
\end{aligned}
$$


Note that the right-hand side of (3.20) is bounded as $y \rightarrow z$.

When $y \rightarrow z$, we observe that $O(|x-y|)$ is small, for $x \in D^{(1)} \cap B_{z}$. Hence, by formulas (2.11)-(2.12) for the principal parts of the fundamental solutions of the second-order elliptic differential equations with discontinuous coefficients we obtain

$$
\left|\int_{D^{(1)} \cap B_{z}} k_{0}^{2} q_{1}(x) w_{1}(x, y) w_{2}(x, y) d x\right| \leq c_{1},
$$

and, using formulas (2.11)-(2.12) again, we get:

$$
\int_{D^{(1)} \cap B_{z}} a_{1}(x) \operatorname{grad} w_{1}(x, y) \cdot \operatorname{grad} w_{2}(x, y) d x \geq c_{2} \int_{D^{(1)} \cap B_{z}} \frac{d x}{|x-y|^{4}}
$$

where $c_{1}$ and $c_{2}$ are positive constants.

The right-hand side of (3.22) tends to $+\infty$ as $y \rightarrow z$ if $z \in \mathbb{R}^{3}$, and therefore all the terms in (3.20), except one, are bounded. Thus, a contradiction. If the dimension of the space is greater than 3 , the conclusion of Theorem 3.1 remains valid and the argument needs only a slight modification.

Hence $D^{(1)}$ must be contained in $D^{(2)}$. Arguing similarly we obtain that $D^{(2)}$ must be contained in $D^{(1)}$, and therefore that $D^{(1)}=D^{(2)}$. Hence $S_{0}^{(1)}$ coincides with $S_{0}^{(2)}$. Now, we follow the above procedure stepwise; repeating it, we conclude that $S_{m}^{(1)}$ coincides with $S_{m}^{(2)}$ for all $m=0,1, \ldots, M$, q.e.d.

Remark 3.1 The previous result and proof remain valid for variable coefficients $a, q$ belonging to the class of piecewise-smooth functions with a non-zero jump at $S_{m}$. Moreover, our argument shows that $a_{1}=a_{2}$ at $S_{m}$ even if one does not assume a priori that $a_{1}=a_{2}$ in the layers.

If $a(x)$ in (3.1) is $H^{2}$-smooth, rather than piecewise- $H^{2}$-smooth, then it was proved in [9] (see also [12]) that the scattering data at two frequencies determine both $a(x)$ and $q(x)$ in (3.1) uniquely. Stability of the solution to the inverse obstacle problem was studied in [6], [13] and [14].

\section{References}

[1] C. Athanasiadis - I.G. Stratis: Low frequency acoustic scattering by an infinitely stratified scatterer, Rend. Mat. Appl., 15, 1995, 133-152.

[2] C. Athanasiadis - I.G. Stratis: On some elliptic transmission problems, Ann. Polon. Math., 63, 1996, 137-154.

[3] R. Dautray - J.-L. Lions: Mathematical Analysis and Numerical Methods for Science and Technology, Vol. 4: Integral Equations and Numerical Methods, Springer, Berlin, 1990. 
[4] S.M.Kozlov: Asymptotics of fundamental solutions of divergent-type second order differential equations, Matem. Sbornik, 113 (155), N2, (1980), 302-323; English transl. in Math. USSR Sb., 41, 1982, 249-267.

[5] C. Miranda: Partial Differential Equations of Elliptic Type, Springer, New York, 1970.

[6] A.G. Ramm: Scattering by Obstacles, Reidel, Dordrecht, 1986.

[7] A.G. Ramm: Completeness of the products of solutions to PDE and uniqueness theorems in inverse scattering, Inverse Problems, 3, 1987, 77-82.

[8] A.G. Ramm: A uniqueness theorem for a boundary inverse problem, Inverse Problems, 4, 1988, 1-5.

[9] A.G. Ramm: Multidimensional inverse problems and completeness of the products of solutions to PDE, J. Math. Anal. Appl., 134, 1988, 211253.

[10] A.G. Ramm: Multidimensional inverse problems: uniqueness theorems, Appl. Math. Lett., 1, 1988, 377-380.

[11] A.G. Ramm: Completeness of the products of solutions of PDE and inverse problems, Inverse Problems, 6, 1990, 643-664.

[12] A.G. Ramm: Multidimensional Inverse Scattering Problems, Longman Scientific \& Wiley, New York, 1992.

[13] A.G. Ramm: Stability of the solution to inverse obstacle scattering problem, J. Inverse and Ill-Posed Problems, 2, 1994, 269-275.

[14] A.G. Ramm: Stability estimates for obstacle scattering, J. Math. Anal. Appl., 188, 1994, 743-751.

[15] A.G. Ramm: Uniqueness theorems for inverse obstacle scattering problems in Lipschitz domains, Applicable Analysis, 59, 1995, 377-383.

[16] A.G. Ramm: Existence and uniqueness of scattering solutions in nonsmooth domains, J. Math. Anal. Appl., 201, 1996, 329-338.

[17] A.G. Ramm: Fundamental solutions to some elliptic equations with discontinuous senior coefficients, preprint, 1997.

[18] P.C. Sabatier: On the scattering by discontinuous media, in Inverse Problems in Partial Differential Equations, (D. Colton, R. Ewing, W. Rundell eds.), SIAM, Philadelphia, 1990, 85-100. 